\title{
Magnetic Flux Lines in Complex Geometry Type-II Superconductors Studied by the Time Dependent Ginzburg-Landau Equation.
}

\author{
Alstrøm, Tommy Sonne; Sørensen, Mads Peter; Pedersen, Niels Falsig; Madsen, Søren
}

Published in:

Acta Applicandae Mathematicae

Link to article, DOI:

$10.1007 / \mathrm{s} 10440-010-9580-8$

Publication date:

2010

Document Version

Publisher's PDF, also known as Version of record

Link back to DTU Orbit

Citation (APA):

Alstrøm, T. S., Sørensen, M. P., Pedersen, N. F., \& Madsen, S. (2010). Magnetic Flux Lines in Complex Geometry Type-II Superconductors Studied by the Time Dependent Ginzburg-Landau Equation. Acta Applicandae Mathematicae, 115(1), 63-74. https://doi.org/10.1007/s10440-010-9580-8

\section{General rights}

Copyright and moral rights for the publications made accessible in the public portal are retained by the authors and/or other copyright owners and it is a condition of accessing publications that users recognise and abide by the legal requirements associated with these rights.

- Users may download and print one copy of any publication from the public portal for the purpose of private study or research.

- You may not further distribute the material or use it for any profit-making activity or commercial gain

- You may freely distribute the URL identifying the publication in the public portal 


\title{
Magnetic Flux Lines in Complex Geometry Type-II Superconductors Studied by the Time Dependent Ginzburg-Landau Equation
}

\author{
Tommy Sonne Alstrøm • Mads Peter Sørensen • \\ Niels Falsig Pedersen • Søren Madsen
}

Received: 15 April 2010 / Accepted: 20 July 2010

(C) Springer Science+Business Media B.V. 2010

\begin{abstract}
The time-dependent Ginzburg-Landau equation is solved numerically for type-II superconductors of complex geometry using the finite element method. The geometry has a marked influence on the magnetic vortex distribution and the vortex dynamics. We have observed generation of giant vortices at boundary defects, suppressing the superconducting state far into the superconductor.
\end{abstract}

Keywords Type II superconductivity · Ginzburg-Landau equation · Vortex lattices · Giant vortices

\section{Introduction}

In 1950 V.L. Ginzburg and L.D. Landau proposed a phenomenological theory for superconducting phase transitions [1]. The theory is based on a Schrödinger equation with a $\phi-4$ potential and a kinetic term involving the momentum operator. For type-II superconductors the Ginzburg-Landau equation models the magnetic field penetration through quantized current vortices as the externally applied magnetic field exceeds a threshold value. A number of variants of the Ginzburg-Landau equation has been used to investigate pattern formation

This paper is dedicated to the memory of Alwyn C. Scott.

T.S. Alstrøm

Department of Informatics, Technical University of Denmark, 2800 Kongens Lyngby, Denmark

e-mail: tsal@imm.dtu.dk

M.P. Sørensen $(\varangle) \cdot$ N.F. Pedersen

Department of Mathematics, Technical University of Denmark, 2800 Kongens Lyngby, Denmark e-mail: M.P.Soerensen@mat.dtu.dk

S. Madsen

Mads Clausen Institute, University of Southern Denmark, Alsion 2, 6400 Sønderborg, Denmark

e-mail: sma@mci.sdu.dk

N.F. Pedersen

e-mail: N.F.Pedersen@mat.dtu.dk

Published online: 14 August 2010 
in various nonlinear media, not only in superconductivity. Several review articles in the Encyclopedia of Nonlinear Science by A.C. Scott [2] provides a number of these variants and their application in nonlinear science.

In this article our focus is on solving the time dependent Ginzburg-Landau equation [3, 4] coupled to a magnetic field for type II superconductors of complex geometry. The model consists of four time-dependent and coupled nonlinear partial differential equations. Various numerical methods have been developed for the Ginzburg-Landau equation. A review is presented by $\mathrm{Du}$ [5] of the finite difference, the finite element and the finite volume methods. Emphasis is on large scale computations of vortex dynamics in tree dimensions. Spatial discretization using the Galerkin finite element method is used by $\mathrm{Mu}$ [6] in combination with a Crank-Nicolson implicit time integration scheme for the time dependent Ginzburg-Landau equation. A finite difference scheme with explicit, semi implicit, linear and nonlinear semi implicit time integrations have been investigated and compared by Gunter et al. [7]. Even though the finite element method has been used by $\mathrm{Du}$ [5] and $\mathrm{Mu}$ [6], these authors never utilized the finite element method to solve problems with more complicated geometries than squares, cubes, circles and spheres. Our goal is to take up the finite element method to investigate vortex penetration and dynamics into superconductors of more complex geometry. The finite element method is particularly well suited for handling complex shapes of the superconductors.

The geometry of the superconductor has a marked influence on the vortex distribution and dynamics. Previous investigations show that the spatial extent of a mesoscopic superconductor influences the formation of the superconducting state [8-10]. Internal bulk properties can likewise lead to more complicated states as in two-band superconductors described by a two-component Ginzburg-Landau model [11]. In a number of articles the influence of surface defects has been investigated [12-14]. For a regular superconducting disk, the energy barrier has been calculated by Berdiyorov et al. [10] for a single vortex to enter or exit the disk. The calculation has been performed using the static Ginzburg-Landau model and compared to the London theory. In the references $[12,13]$ Peeters, Baelus and Schweigert have found that surface defects on a superconducting disk lead to lowering of the energy barrier experienced by vortices entering the superconducting disk. This means that the vortices start entering a superconductor with a surface defect for lower values of the externally applied magnetic field. For type II superconductors the first critical magnetic field decreases in the presence of a defect. The magnetization curve for a superconductor with a defect is quantitatively changed [14]. Vodolazov et al. [14] investigate the time dependent Ginzburg-Landau model for a rectangular shaped superconductor with a geometric defect and a material defect at the surface. They are able to provide a criterion for vortex penetration at the defects based on a critical value of the supervelocity $\nabla \phi-\mathbf{A}$, where $\phi$ is the phase of the GinzburgLandau parameter and $\mathbf{A}$ is the magnetic vector potential. For a certain critical value of the magnetic field, vortices start entering the superconductor at the defects. They also observe formation of giant vortices at the defects, enclosing several flux quanta.

The purpose of our study is to illustrate the dynamics of vortices entering a defect in a circular shaped type-II superconductor with a geometric defect in the form of a triangular indent. The static investigations in $[12,13]$ tell that vortices must enter at defects which lower the barrier energy. However, the dynamics of entering vortices lacks investigation. The dynamic features have not been presented in details by Vodolazov et al. in Ref. [14]. Therefore the purpose here is to illustrated these dynamic features by simulations of the time dependent Ginzburg-Landau equations. Similarly to [14] we have observed generation of giant vortices at boundary defects, suppressing the superconducting state far into the superconductor. These giant vortex areas with suppressed superconductivity sheds off magnetic 
vortices until an equilibrium is obtained between the distributed single vortices and the giant vortex area. Giant vortex regions can also appear around pinning sites modelled by spatial inhomogeneous potentials [15] and they have been predicted in the center of a hollow cylinder [16] and in disks [17]. In square shaped superconductors it has been demonstrated that the time evolution of vortices can lead to nucleation into multi vortex states or giant vortex formation [18]. The single vortices in type II superconductors are stable nonlinear coherent structures, which share properties with the soliton vortices observed in long Josephson junctions and thoroughly investigated in the seminal paper by Scott and McLaughlin [19].

\section{The Time Dependent Ginzburg-Landau Equation}

The time dependent Ginzburg-Landau equation is a phenomenological model for the dynamics of the superconducting state. The order parameter is denoted by $\Psi=\Psi(x, y, z, t)$ and it can be interpreted as the density of superconducting charge carriers (Cooper pairs) at position $(x, y, z)$ and at time $t$. Strictly $\Psi$ also depends on the absolute temperature $T$, however, this dependence is omitted from the list of arguments. Below the critical temperature $T_{c}$ we are in the superconducting state with $|\Psi|>0$, and above the critical temperature the order parameter vanishes. The Gibbs energy of the superconducting state is $G_{s}=G_{n}-\alpha|\Psi|^{2}+(\beta / 2)|\Psi|^{4}$, where $G_{n}$ is the Gibbs energy for the normal conducting state. $\beta$ is assumed constant but $\alpha$ is taken to be related to the temperature through $\alpha(T)=\alpha(0)\left(1-T / T_{c}\right)$. The sign shift of $\alpha$ guarantees the phase transition from the normal state to the superconducting state as the temperature is decreased from a value above $T_{c}$ to a value below $T_{c}$. For a type II superconductor the time-dependent Ginzburg-Landau equations coupled to a penetrating magnetic field $\mathbf{B}_{\mathbf{i}}=\nabla \times \mathbf{A}$, with $\mathbf{A}$ being the magnetic vector potential, reads in SI units [4]

$$
\begin{aligned}
\frac{\hbar^{2}}{2 m D}\left(\frac{\partial}{\partial t}+i \frac{q}{\hbar} \Phi\right) \Psi= & -\frac{1}{2 m}\left(\frac{\hbar}{i} \nabla-q \mathbf{A}\right)^{2} \Psi+\alpha \Psi-\beta|\Psi|^{2} \Psi \\
\sigma\left(\frac{\partial \mathbf{A}}{\partial t}+\nabla \Phi\right)= & \frac{q \hbar}{2 m i}\left(\Psi^{*} \nabla \Psi-\Psi \nabla \Psi^{*}\right) \\
& -\frac{q^{2}}{m}|\Psi|^{2} \mathbf{A}-\frac{1}{\mu_{0}} \nabla \times \nabla \times \mathbf{A}
\end{aligned}
$$

As usual the parameter $\hbar$ is Planck's constant divided by $2 \pi$ and $i$ is the imaginary number $\sqrt{-1}$. The charge of a Cooper pair is denoted $q=2 e$ and the Cooper pair mass is $m$. The parameter $D$ is a phenomenological diffusion coefficient [3]. The second equation (2) describes the dynamics of the magnetic field coupled to the Ginzburg-Landau equation (1). Here $\Phi=\Phi(x, y, z, t)$ is the electric potential and $\sigma$ is the conductivity of the normal (non superconducting) current. The parameter $\mu_{0}$ is the permeability of the free space. To obtain (2) it is assumed that the external applied magnetic field $\mathbf{B}_{\mathbf{a}}$ is a uniform static field, which formally means that the curl of $\mathbf{B}_{\mathbf{a}}$ vanishes. If this is not the case the term $\nabla \times \mathbf{B}_{\mathbf{a}} / \mu_{0}$ needs to be added to the right hand side of (2).

In order to solve the time dependent Ginzburg-Landau equation we need to specify appropriate boundary conditions of the superconducting sample. Those are [5]

$$
\left(\frac{\hbar}{i} \nabla \Psi-q \mathbf{A} \Psi\right) \cdot \mathbf{n}=0, \quad \text { on } \partial \Omega,
$$




$$
\begin{aligned}
& \mathbf{B}_{\mathbf{i}}=\mathbf{B}_{\mathbf{a}}, \quad \text { on } \partial \Omega, \\
& \left(\frac{\partial \mathbf{A}}{\partial t}+\nabla \Phi\right) \cdot \mathbf{n}=0, \quad \text { on } \partial \Omega .
\end{aligned}
$$

on the boundary $\partial \Omega$ of the superconducting domain $\Omega$. The outward normal vector to the boundary is denoted by $\mathbf{n}$. An important property of the time-dependent model is that the current density $\mathbf{J}$ is

$$
\mathbf{J}=\sigma \mathbf{E}+\mathbf{J}_{s},
$$

where $\mathbf{J}_{s}$ is the supercurrent and $\mathbf{E}$ is the electric field given by

$$
\mathbf{E}=-\frac{\partial \mathbf{A}}{\partial t}-\nabla \Phi
$$

The last equation in (3) is easily justified as the normal current is parallel to the electric field, and hence $\mathbf{E} \cdot \mathbf{n}=0$ on the boundary as the current do not pass across the boundary of the superconductor. The time-dependent Ginzburg-Landau model is valid for temperatures close to $T_{c}$ and the theory also requires the superconductor to be gap less [3, 20].

\section{Normalization and Gauge Invariance}

We can scale the space coordinates according to the London penetration depth $\lambda$ of the magnetic field or according to the Ginzburg-Landau coherence length $\xi=\hbar / \sqrt{2 m \alpha}$. The ratio between the two length scales is the Ginzburg-Landau parameter $\kappa=\lambda / \xi$. Here we choose to scale with respect to $\lambda$ and with the dimensionless variables marked by primes, (1) and (2) can be transformed through

$$
\begin{aligned}
& (x, y, z, t)=\left(\lambda x^{\prime}, \lambda y^{\prime}, \lambda z^{\prime}, \frac{\xi^{2}}{D} t^{\prime}\right), \quad \mathbf{A}=\frac{\hbar}{q \xi} \mathbf{A}^{\prime}, \\
& \Psi=\sqrt{\frac{\alpha}{\beta}} \Psi^{\prime}, \quad \Phi=\alpha D \kappa^{2} \sqrt{\frac{2 \mu_{0}}{b}} \Phi^{\prime}, \quad \sigma=\frac{1}{\mu_{0} D \kappa^{2}} \sigma^{\prime} .
\end{aligned}
$$

Going through the algebra and omitting the prime on the dimensionless variables, the scaling results in the normalized Ginzburg-Landau equations

$$
\begin{aligned}
& \left(\frac{\partial}{\partial t}+i \kappa \Phi\right) \Psi=-\left(\frac{i}{\kappa} \nabla+\mathbf{A}\right)^{2} \Psi+\Psi-|\Psi|^{2} \Psi \\
& \sigma\left(\frac{\partial \mathbf{A}}{\partial t}+\nabla \Phi\right)=\frac{1}{2 i \kappa}\left(\Psi^{*} \nabla \Psi-\Psi \nabla \Psi^{*}\right)-|\Psi|^{2} \mathbf{A}-\nabla \times \nabla \times \mathbf{A} .
\end{aligned}
$$

By this procedure we are left with only two parameters, the Ginzburg-Landau parameter $\kappa$ and the normalized conductivity $\sigma$. The boundary conditions (3) are transformed into

$$
\begin{aligned}
& \left(\frac{\hbar}{i} \nabla \Psi+\mathbf{A} \Psi\right) \cdot \mathbf{n}=0, \quad \text { on } \partial \Omega, \\
& \nabla \times \mathbf{A}=\mathbf{B}_{\mathbf{a}}, \quad \text { on } \partial \Omega, \\
& \left(\frac{\partial \mathbf{A}}{\partial t}+\nabla \Phi\right) \cdot \mathbf{n}=0, \quad \text { on } \partial \Omega .
\end{aligned}
$$


We shall keep in mind that the none primed variables now are the scaled dimensionless variables.

The time dependent Ginzburg-Landau equations have the property of gauge invariance. Given a function $\chi(x, y, z, t)$, the gauge transformation is defined as

$$
\tilde{\Psi}=\Psi e^{i \kappa \chi}, \quad \tilde{\mathbf{A}}=\mathbf{A}+\nabla \chi, \quad \tilde{\Phi}=\Phi-\frac{\partial \chi}{\partial t} .
$$

In order to obtain well posed equations we need to fix the gauge [20, 21]. The most convenient choice turns out to be the zero electric potential gauge where $\tilde{\Phi}$ is chosen to be zero. From the transformation given in (10) we then have

$$
\frac{\partial \chi}{\partial t}=\Phi
$$

With vanishing $\tilde{\Phi}$ the last equation in the boundary conditions (9) results in

$$
\frac{\partial \mathbf{A}}{\partial t} \cdot \mathbf{n}=0
$$

This equation can be integrated leading to $\mathbf{A} \cdot \mathbf{n}=0$. Omitting the tildes and with the above gauge transformation our final version of the Ginzburg-Landau equations becomes

$$
\begin{aligned}
\frac{\partial \Psi}{\partial t} & =-\left(\frac{i}{\kappa} \nabla+\mathbf{A}\right)^{2} \Psi+\Psi-|\Psi|^{2} \Psi, \\
\sigma \frac{\partial \mathbf{A}}{\partial t} & =\frac{1}{2 i \kappa}\left(\Psi^{*} \nabla \Psi-\Psi \nabla \Psi^{*}\right)-|\Psi|^{2} \mathbf{A}-\nabla \times \nabla \times \mathbf{A} .
\end{aligned}
$$

with the boundary conditions

$$
\begin{aligned}
& \nabla \Psi \cdot \mathbf{n}=0, \quad \text { on } \partial \Omega, \\
& \nabla \times \mathbf{A}=\mathbf{B}_{\mathbf{a}}, \quad \text { on } \partial \Omega, \\
& \mathbf{A} \cdot \mathbf{n}=0, \quad \text { on } \partial \Omega .
\end{aligned}
$$

The partial differential equations (13) and (14) are solved numerically for various geometries $\Omega \subset \mathcal{R}^{2}$ in the two dimensional space. As we wish to investigate relatively complex shapes of the superconductor, we have chosen to use a finite element method with the simplified boundary conditions in (15) for various external applied magnetic field strength $\mathbf{B}_{a}=\left(0,0, B_{a z}\right)$ in the $z$-direction. The initial conditions are $|\Psi|=1$ corresponding to the Meissner state and zero magnetic field inside the superconductor. The time-dependent Ginzburg-Landau model has been implemented in the finite element programme COMSOL [22] using quadratic Lagrange elements, which turned out to give sufficiently accurate numerical solutions.

\section{Numerical Simulations}

In this section we present numerical simulations of the dynamics of the magnetic vortices in type II superconductors for a circular disk with a triangular defect or indent, see Fig. 1. The (13) and (14) are solved in the two dimensional space $(x, y) \in \Omega$, with $\Phi=\Phi(x, y, t)$ 

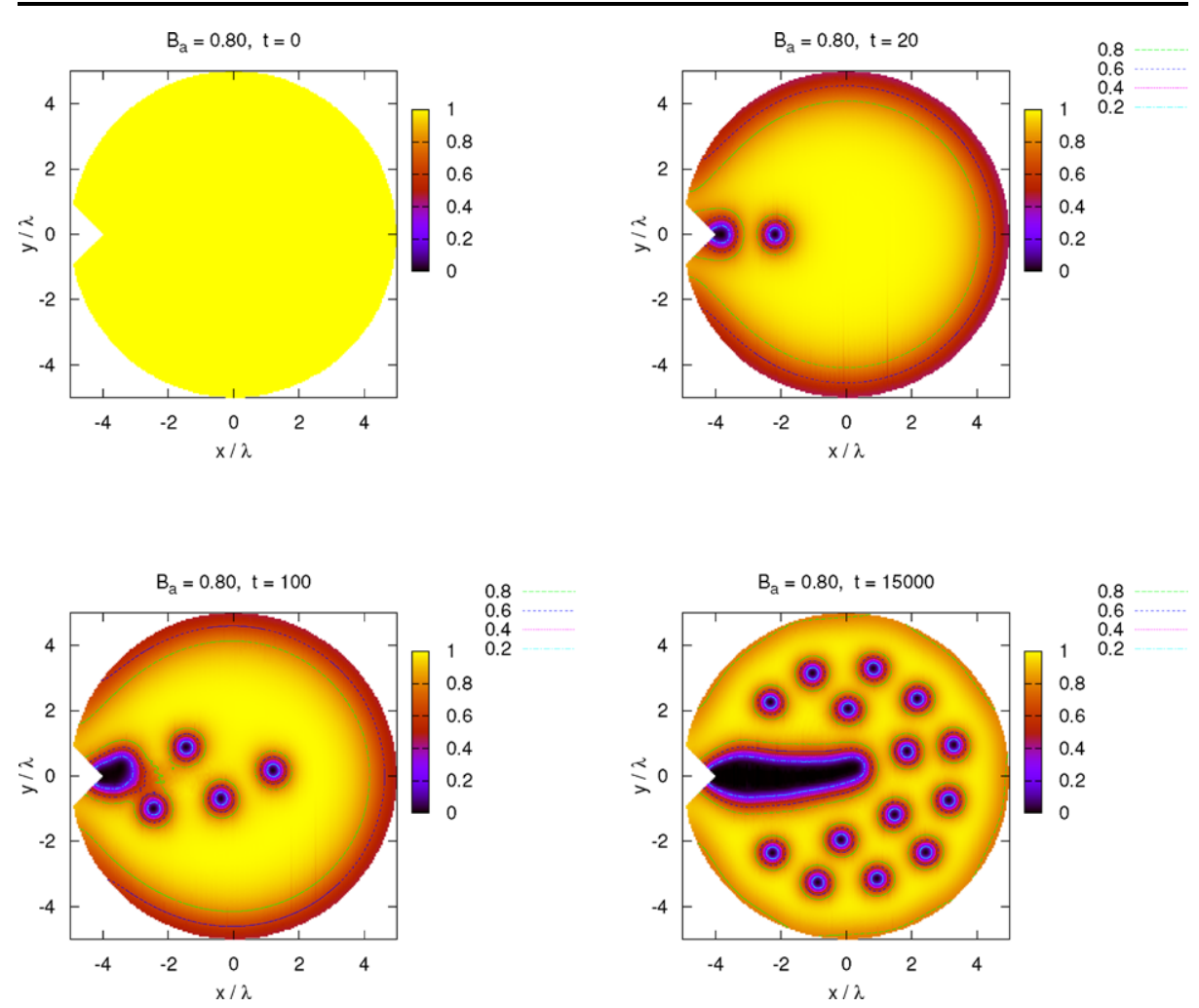

Fig. 1 The absolute value $|\psi(x, y, t)|$ of the order parameter for $\kappa=4$ and $B_{a z}=0.80$ at times: $t=0,20$, 100,15000

and the vector potential given in component form $\mathbf{A}(x, y, t)=\left(A_{x}(x, y, t), A_{y}(x, y, t)\right)$. The normalized magnetic field $\mathbf{B}_{i}=\nabla \times \mathbf{A}$ is along the $z$-axis direction. In the computer implementation the order parameter has been split into its real and imaginary parts and writing the vector field A in component form, (13) and (14) become four coupled partial differential equations. The numerical results for a circular disk with a boundary defect are presented in Figs. 1 and 2. In Fig. 1 we use an applied magnetic field of strength $B_{a z}=0.8$ and the Ginzburg-Landau parameter $\kappa=4$. In this case the energy barrier at the boundary is lowest at the triangular defect and therefore all vortices enter the superconductor through this defect. Our main goal is to illustrate the dynamics of the magnetic flux entering at the defect. Initially three magnetic vortices enter the superconductor in a straight line at the tip of the indent. At the same time a normal conducting state is formed at the defect tip enclosing several elementary flux quanta. This multiple vortex state growths in size and more single vortices shed off the giant vortex in an upward and downward succession. This later dynamics is like the von Karmann vortices in the wake of a fluid flow after a circular rod. Eventually, the dynamics settles into a pattern with a large in going normal state or giant vortex. ${ }^{1}$

\footnotetext{
${ }^{1}$ The associated animations are found at: http://www2.mat.dtu.dk/people/M.P.Soerensen/animations/ Ginzburg-Landau.
} 

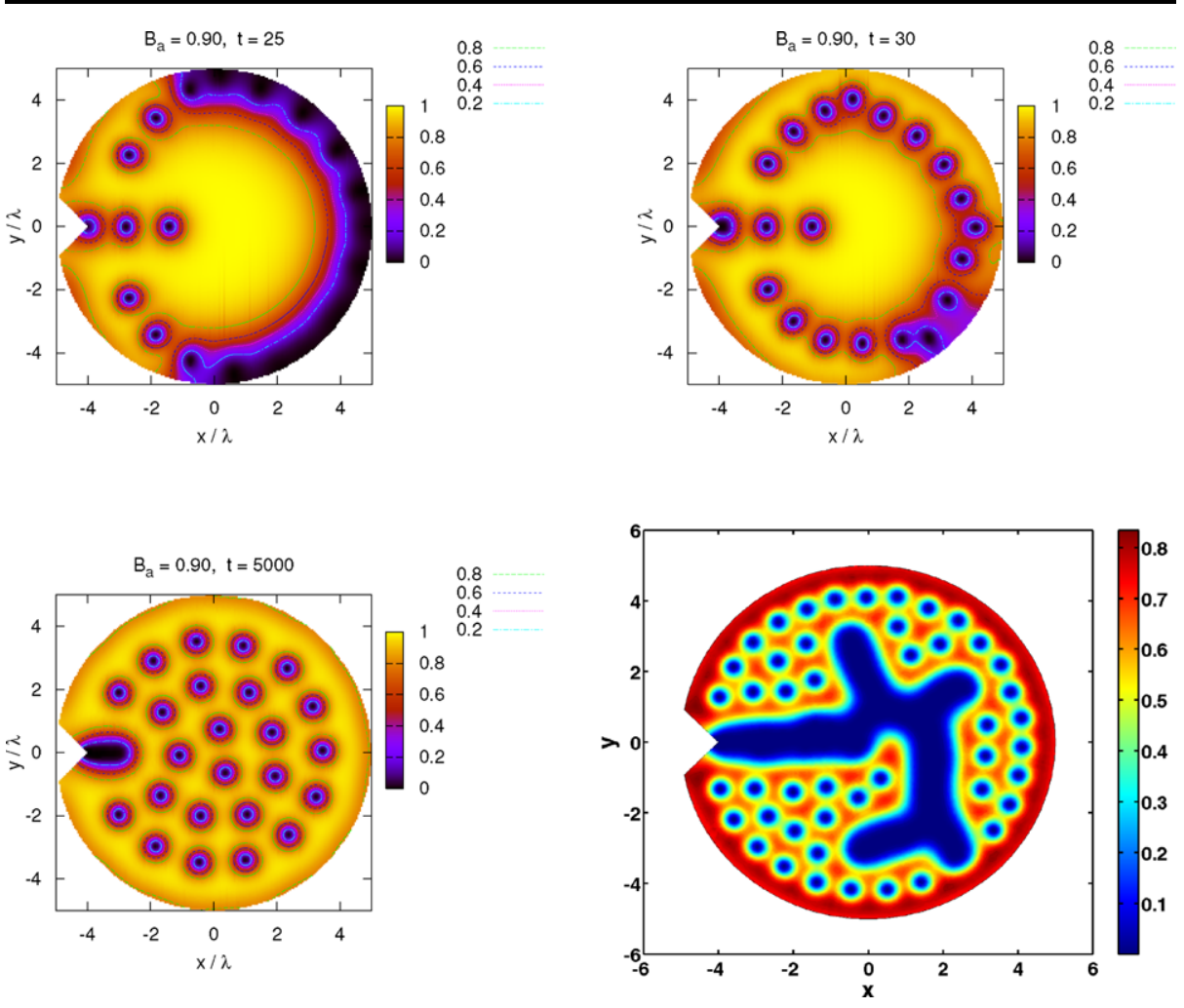

Fig. 2 The absolute value $|\psi(x, y, t)|$ of the order parameter for $\kappa=4$ and $B_{a z}=0.90$ at times: $t=20,25$, and 5000. The lower right subfigure shows the absolute value of $|\psi(x, y, t)|$ for $\kappa=4$ and $B_{a z}=2.02$ at stationary state

Further increase of the magnetic field strength to $B_{a z}=0.9$ leads to the dynamics shown in Fig. 2. Vortices enter again at the triangular indent but now the magnetic field is strong enough to enter through the disk boundary, even though the barrier energy is higher here than at the defect. As a result this leads to depletion of the size of the normal conducting region at the triangular indent, illustrated in the lower left subplot of Fig. 2. Increasing the magnetic field in small increments and simulating to stationary state leads to more complex pattern formation with a fingering structure of the giant vortex. This phenomenon is displayed in the lower right subfigure in Fig. 2, where $B_{a z}=2.02$. For values of $B_{a z}$ exceeding 3.8 we observed no traces of penetrating vortices, meaning that the critical magnetic field is $B_{\text {critical }}=3.8$.

\section{Energy Patterns in the Superconductor}

The total energy density $\mathcal{H}_{\text {tot }}=\mathcal{H}_{\text {tot }}(x, y, t)$ of the superconductor can be split into a sum of three parts, the superconducting energy density $\mathcal{H}_{\text {sup }}=\mathcal{H}_{\text {sup }}(x, y, t)$, the magnetic energy density $\mathcal{H}_{\text {mag }}=\mathcal{H}_{\text {mag }}(x, y, t)$ and finally the interaction energy density $\mathcal{H}_{\text {int }}=\mathcal{H}_{\text {int }}(x, y, t)$. 

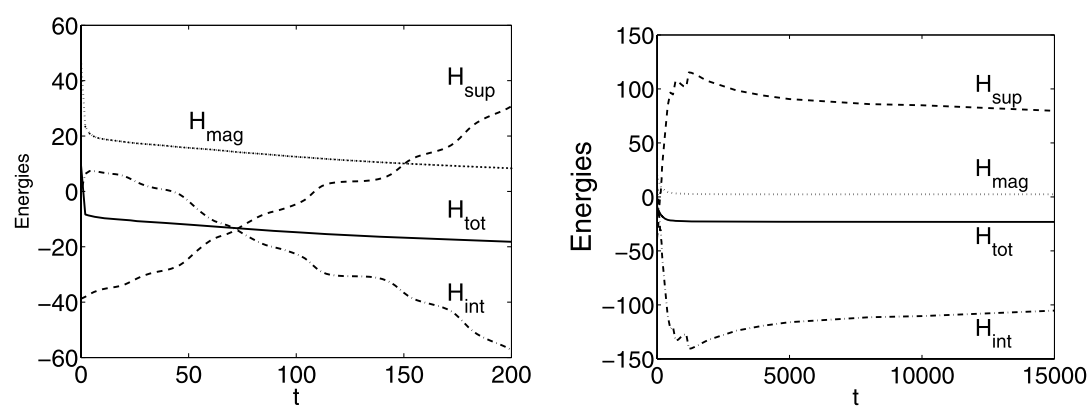

Fig. 3 The total energies as function of time $t$ for the circular disk with a defect. Parameter values are $\kappa=4$, $B=0.8$ and $\sigma=1$. The total energy $H_{\text {tot }}$ (solid line), the total superconducting energy $H_{\text {sup }}$ (dashed line), the total magnetic energy $H_{\text {mag }}$ (dotted line), the total interaction energy $H_{\text {int }}$ (dash-dotted line)

These energies are given by

$$
\begin{aligned}
\mathcal{H}_{\text {sup }} & =\frac{1}{\kappa^{2}}|\nabla \psi|^{2}-|\psi|^{2}+\frac{1}{2}|\psi|^{4}, \\
\mathcal{H}_{\text {mag }} & =\left(\mathbf{B}_{a}-\nabla \times \mathbf{A}\right)^{2}, \\
\mathcal{H}_{\text {int }} & =\frac{i}{\kappa} \mathbf{A}\left((\nabla \psi) \psi^{*}-\psi\left(\nabla \psi^{*}\right)\right)+|\mathbf{A}|^{2}|\psi|^{2} .
\end{aligned}
$$

The total energy density is $\mathcal{H}_{\text {tot }}=\mathcal{H}_{\text {sup }}+\mathcal{H}_{\text {mag }}+\mathcal{H}_{\text {int }}$. By integrating the above three energies over the entire space $\Omega$, i.e. $H_{\text {tot }}=\int_{\Omega} \mathcal{H}_{\text {tot }}(x, y, t) d x d y$ and similarly for the superconducting, magnetic and the interaction energies, we obtain the results shown in Fig. 3, where the left hand figure shows the energies as function of time $t$ for $0 \leq t \leq 200$, and the right hand figure shows the same energies for $0 \leq t \leq 15,000$. The initial condition corresponds to the Meissner state, which here is an out of equilibrium state, and hence the total energy decreases to its steady state value as the magnetic flux lines enter the superconductor through the triangular defect. The magnetic energy decreases also. As more magnetic flux lines enter, the superconducting energy state increases in clearly visible jumps. Each jump corresponds to one flux line entering the superconductor. The interaction energy decreases and also here we observe a clear jump in the energy decrease as fluxons enter the superconductor. These jumps are also present in the total energy and the magnetic energy, but they a very small and hardly observable. After all the flux lines have entered the superconductor, the interaction energy and the superconducting energy settle slowly to their steady state values by rearranging the flux line positions in the superconductor.

In Fig. 4 we show the total energy density and the energy density of the superconducting state at time $t=100$. As expected the energy of the superconducting state is elevated at the triangular indent, however, the superconducting energy is also slightly elevated along the traces of the 4 entering vortices. Along these traces the phase is changing rapidly in the superconducting state, and this leads to enhanced superconducting energy due to the first term in (16). In Fig. 5a we depict the magnetization energy $\mathcal{H}_{\text {mag }}(x, y, 100)$ as function of space at time $t=100$ and Fig. 5b shows the interaction energy $\mathcal{H}_{\text {int }}(x, y, 100)$. The interaction energy is lowered considerable in the regions where both the magnetic field and the order parameter change and hence the formation of penetrating flux is favored. The phase $\phi$ at time $t=100$, defined as the argument of $\psi$, is shown in Fig. 6, where $-\pi<\phi<\pi$. 

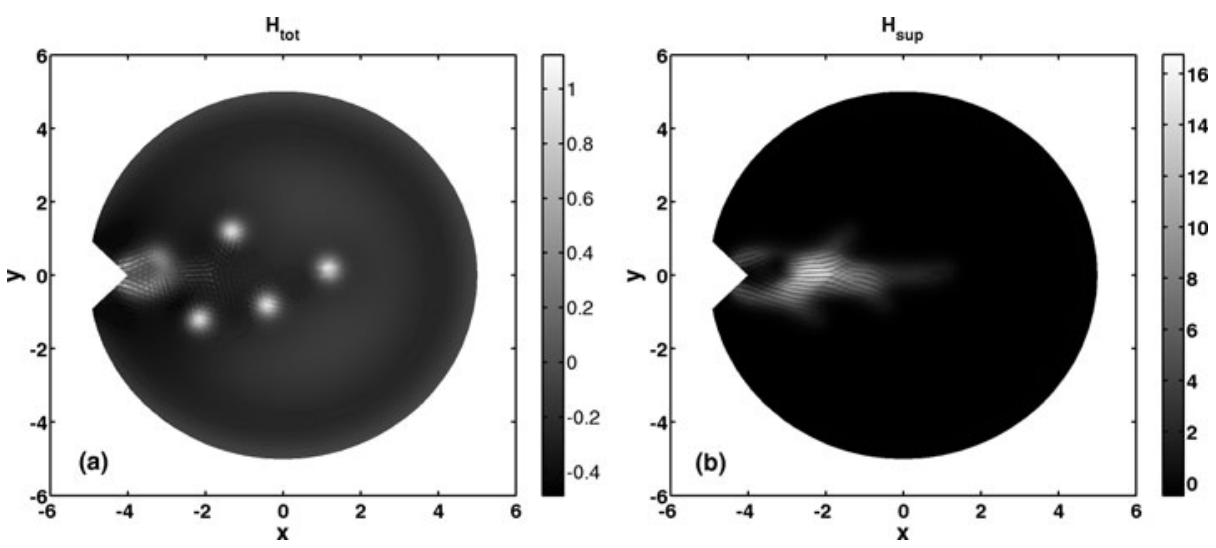

Fig. 4 (a) The total energy density $\mathcal{H}_{\text {tot }}(x, y, 100)$ as function of space at time $t=100$. (b) The total superconducting energy density $\mathcal{H}_{\text {sup }}(x, y, 100)$ as function of space at time $t=100$. The parameter values are $\kappa=4, B=0.8$ and $\sigma=1$
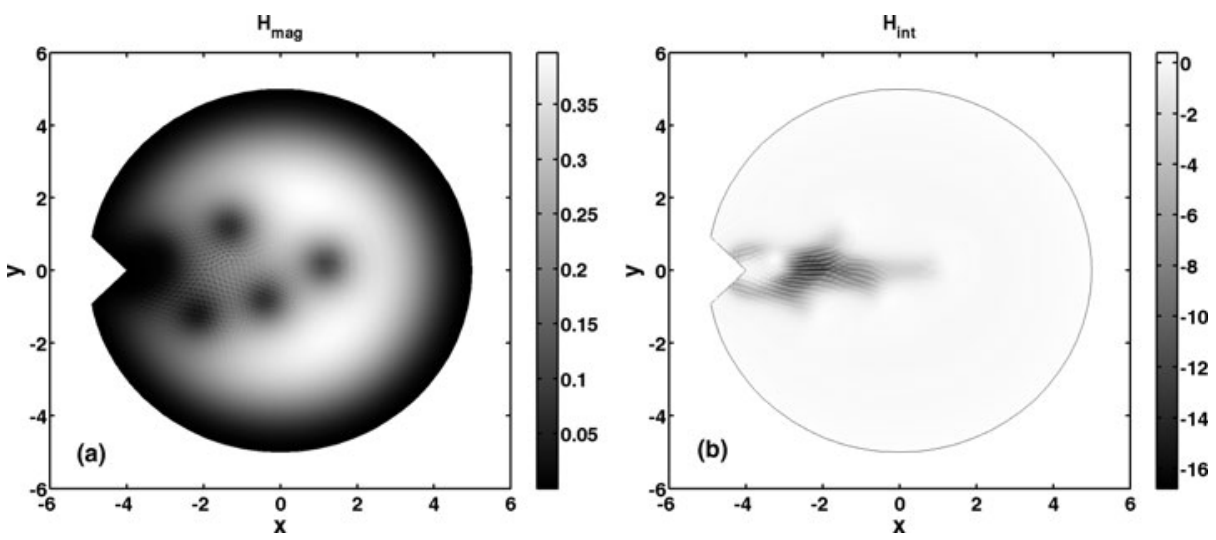

Fig. 5 (a) The magnetization energy density $\mathcal{H}_{\text {mag }}(x, y, 100)$ as function of space at time $t=100$. (b) The interaction energy density $\mathcal{H}_{\text {int }}(x, y, 100)$ as function of space at time $t=100$. The parameter values are $\kappa=4, B=0.8$ and $\sigma=1$

An interesting feature is that the phase pattern clearly indicates the path along which the magnetic fluxes penetrates into the superconductor.

\section{Summary}

The time-dependent Ginzburg-Landau equations have been solved numerically with a finite element algorithm for superconductors of complex geometries in two space dimensions. For given applied magnetic fields we have computed the dynamical behavior of the penetrating magnetic vortices into a type two superconductor and illustrated their final pattern formation.

Our main result is the finding of the formation of a giant vortex at a sharp concave indent penetrating into circular shaped superconductor. Initially, the giant vortex shed off single quantum flux lines which distribute equally throughout the superconductor. The giant 
Fig. 6 The phase as function of space at time $t=100$. The parameter values are $\kappa=4$, $B=0.8$ and $\sigma=1$

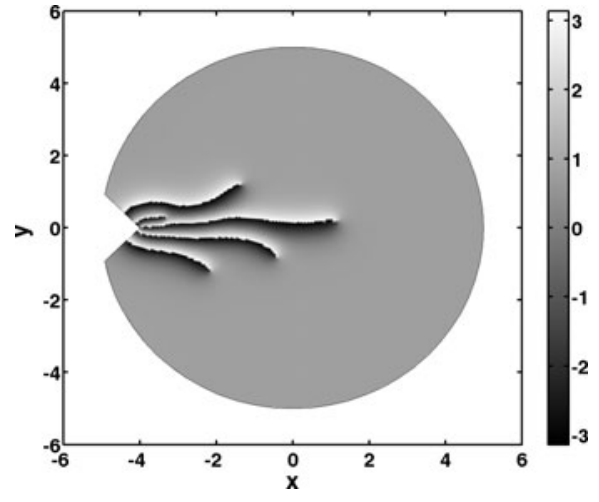

vortex develops into an elongated structure and coexist with the single quantum magnetic fluxes. However, higher magnetic fields leading to flux penetration at boundaries away from the indent can suppress the formation of the elongated giant vortex and reduce its size considerably.

The calculated energy patterns show that the dynamics of penetrating magnetic flux lines lead to a substantial decrease of the interaction energy, compensating for the relatively strong increase in the superconducting energy in those regions where magnetic flux penetrates into the superconductor.

Acknowledgements We acknowledge financial support from the Danish Natural Science Research Council through project No. 21-02-0500 (MIDIT).

\section{Appendix A}

The numerical simulations are based on a finite element implementation of the GinzburgLandau equations (13)-(14) using the COMSOL Multiphysics software package [23]. The implementation of the boundary conditions (15) turns out not to be completely trivial and hence we discuss the implementation in this appendix. In the implementation we have split the order parameter $\psi(x, y, t)$ into its real part $\operatorname{Re}(\psi)=u_{1}(x, y, t)$ and its imaginary part $\operatorname{Im}(\psi)=u_{2}(x, y, t)$. The magnetic potential $\mathbf{A}$ is written in component form as $\mathbf{A}=\left(u_{3}(x, y, t), u_{4}(x, y, t)\right)$. In order to implement the boundary conditions (15) we have introduced an auxiliary dependent variable $u_{5}(x, y, t)$ for reasons explained below. In general form the COMSOL software package numerically solves the system of partial differential equations

$$
\mathbf{e}_{a} \frac{\partial^{2} \mathbf{u}}{\partial t^{2}}+\mathbf{d}_{a} \frac{\partial \mathbf{u}}{\partial t}+\nabla \cdot \boldsymbol{\Gamma}=\mathbf{F}
$$

In our case the vector function $\mathbf{u}$ equals $\left(u_{1}, u_{2}, u_{3}, u_{4}, u_{5}\right)^{T}$, where " $\mathrm{T}$ " is the transpose. The spatial dimension is $n=2$ and the number of coupled partial differential equations is $N=5$. In general $\mathbf{e}_{a}$ is a $N \times N$ matrix, however, for the current Ginzburg-Landau equations $\mathbf{e}_{a}$ becomes the zero matrix. The matrix $\mathbf{d}_{a}$ is also a $N \times N$ matrix and it may depend on $x, y, t$ and $\mathbf{u}$. The $\boldsymbol{\Gamma}$ is a column vector function of dimension $N$ and each component is a $n$-dimensional column vector function. The right hand side $\mathbf{F}$ in (19) is a $N$-dimensional column vector function. The vectors $\boldsymbol{\Gamma}$ and $\mathbf{F}$ may both depend on $x, y, t, \mathbf{u}, \mathbf{u}_{x}$ and $\mathbf{u}_{y}$, where subscripts denote partial derivatives with respect to the subscript variable. 
In the finite element code, (19) is solved in the weak form and with boundary conditions implemented in implicit form, either as Dirichlet boundary conditions or as Neumann boundary conditions ( [22] and [23]). The two types of conditions can be assigned to different and disjoint parts of the boundary $\partial \Omega$ of the solution space $\Omega$. The Dirichlet boundary condition reads

$$
\mathbf{R}=\mathbf{0} \text { on } \partial \Omega \text {. }
$$

In the above expression the function $\mathbf{R}$ is a vector function of dimension $N$ and each component may depend on $x, y, t, \mathbf{u}, \mathbf{u}_{x}$ and $\mathbf{u}_{y}$. Introducing the outward normal vector $\mathbf{n}$ to $\partial \Omega$, the Neumann boundary condition reads

$$
-n \cdot \boldsymbol{\Gamma}=\mathbf{G} \quad \text { on } \partial \Omega .
$$

The vector function $\mathbf{G}$ of dimension $N$ may depend on $x, y, t, \mathbf{u}, \mathbf{u}_{x}$ and $\mathbf{u}_{y}$. As $\boldsymbol{\Gamma}$ enters the Neumann conditions we are restricted in choosing $\boldsymbol{\Gamma}$ when implementing the GinzburgLandau equations (13)-(14) together with the boundary conditions (15). In our implementation we have chosen to use the Neumann conditions. However, by introducing the auxiliary variable $u_{5}(x, y, t)$ satisfying the equation

$$
\nabla\left[\begin{array}{l}
u_{3} \\
u_{4}
\end{array}\right]=u_{3, x}+u_{4, y}+u_{5},
$$

we can implement the boundary conditions in (15). Here the number of spatial dimensions is $n=2$ and hence $\nabla=\left(\partial_{x}, \partial_{y}\right)$. Subscript $x$ denotes partial differentiation with respect to $x$. Similarly for subscript $y$. The implementation of $\mathbf{d}_{a}$ and $\boldsymbol{\Gamma}$ in COMSOL become

$$
\mathbf{d}_{a}=\left[\begin{array}{lllll}
1 & 0 & 0 & 0 & 0 \\
0 & 1 & 0 & 0 & 0 \\
0 & 0 & \sigma & 0 & 0 \\
0 & 0 & 0 & \sigma & 0 \\
0 & 0 & 0 & 0 & 0
\end{array}\right], \quad \boldsymbol{\Gamma}=\left[\begin{array}{c}
{\left[-u_{1, x} / \kappa^{2},-u_{1, y} / \kappa^{2}\right]^{T}} \\
{\left[-u_{2, x} / \kappa^{2},-u_{2, y} / \kappa^{2}\right]^{T}} \\
{\left[0, u_{4, x}-u_{3, y}-B_{a}\right]^{T}} \\
{\left[-u_{4, x}+u_{3, y}+B_{a}, 0\right]^{T}} \\
{[u 3, u 4]^{T}}
\end{array}\right] .
$$

The right hand side $\mathbf{F}$ contains all other terms and reads

$$
\mathbf{F}=\left[\begin{array}{c}
F_{1} \\
F_{2} \\
\left(u_{1} u_{2, x}-u_{2} u_{1, x}\right) / \kappa-\left(u_{1}^{2}+u_{2}^{2}\right) u_{3} \\
\left(u_{1} u_{2, y}-u_{2} u_{1, y}\right) / \kappa-\left(u_{1}^{2}+u_{2}^{2}\right) u_{4} \\
u_{3, x}+u_{4, y}+u_{5}
\end{array}\right]
$$

where the component $F_{1}=\left(u_{3, x}+u_{4, y}\right) u_{2} / \kappa+2\left(u_{3} u_{2, x}+u_{4} u_{2, y}\right) / \kappa-\left(u_{3}^{2}+u_{4}^{2}\right) u_{1}+u_{1}-$ $\left(u_{1}^{2}+u_{2}^{2}\right) u_{1}$ and the component $F_{2}=-\left(u_{3, x}+u_{4, y}\right) u_{1} / \kappa-2\left(u_{3} u_{1, x}+u_{4} u_{1, y}\right) / \kappa-\left(u_{3}^{2}+\right.$ $\left.u_{4}^{2}\right) u_{2}+u_{2}-\left(u_{1}^{2}+u_{2}^{2}\right) u_{2}$. With the auxiliary equation the boundary conditions (15) now becomes the Neumann condition in (21) with $\mathbf{G}=\mathbf{0}$. Note that the above implementation is not unique, and it is possible to implement the Ginzburg-Landau equations in COMSOL in alternative ways.

\section{References}

1. Ginzburg, V.L., Landau, L.D.: On the theory of superconductivity. Zh. Eksp. Teor. Fiz. 20, 1064 (1950) 
2. Scott, A.C. (Ed.): Encyclopedia of Nonlinear Science. Routledge, New York (2005)

3. Gorkov, L.P., Eliashburg, G.M.: Generalization of the Ginzburg-Landau equations for non-stationary problems in the case of alloys with paramagnetic impurities. Sov. Phys. (JETP) 27, 328 (1968)

4. Gropp, W.D., Kaper, H.G., Leaf, G.K., Levine, D.M., Palumbo, M., Vinokur, V.M.: Numerical simulations of vortex dynamics in Type-II superconductors. J. Comput. Phys. 123, 254-266 (1996)

5. Du, Q.: Numerical approximations of the Ginzburg-Landau models for superconductivity. J. Math. Phys. 46, 095109 (2005)

6. Mu, M.: A linearized Crank-Nicolson-Galerkin method for the Ginzburg-Landau model. SIAM J. Sci. Comput. 18(4), 1028-1039 (1997)

7. Gunter, D.O., Kaper, H.G., Leaf, G.K.: Implicit integration of the time-dependent Ginzburg-Landau equations of superconductivity. SIAM J. Sci. Comput. 23(6), 1943-1958 (2002)

8. Chibotaru, L.F., Ceulemans, A.: Ginzburg-Landau description of confinement and quantization effects in mesoscopic superconductors. J. Math. Phys. 46, 095108 (2005)

9. Geim, A.K., Grigorieva, I.V., Dubonos, S.V., Lok, J.G.S., Maan, J.C., Filippov, A.E., Peeters, F.M., Deo, P.S.: Mesoscopic superconductors as 'artificial atoms' made from Cooper pairs. Physica B 249-251, 445-452 (1998)

10. Berdiyorov, G.R., Cabral, L.R.E., Peeters, F.M.: Surface barrier for flux entry and exit in mesoscopic superconducting systems. J. Math. Phys. 46, 095105 (2005)

11. Madsen, S., Gaididei, Yu.B., Christiansen, P.L., Pedersen, N.F.: Domain walls and textured vortices in a two-component Ginzburg Landau model. Phys. Lett. A 344(4), 432-440 (2005)

12. Baelus, B.J., Peeters, F.M.: The effect of surface defects on the vortex expulsion and penetration in mesoscopic superconducting disks. Physica C 408-410, 543-544 (2004)

13. Peeters, F.M., Schweigert, V.A., Baelus, B.J.: Fractional and negative flux penetration in mesoscopic superconducting disks. Physica C 369, 158-164 (2002)

14. Vodolazov, D.Y., Maksimov, I.L., Brandt, E.H.: Vortex entry conditions in type-II superconductors. Effect of surface defects. Physica C 384, 211-226 (2003)

15. Alama, S., Bronsard, L.: Pinning effects and their breakdown for a Ginzburg Landau model with normal inclusions. J. Math. Phys. 46, 095102 (2005)

16. García, L.C., Giraldo, J.: Giant vortex state in mesoscopic superconductors. Phys. Stat. Sol. (c) 2, 36093612 (2005)

17. Peeters, F.M., Schweigert, V.A., Baelus, B.J., Deo, P.S.: Vortex matter in mesoscopic superconducting disks and rings. Physica C 332, 255-262 (2000)

18. Sardella, E., Malvezzi, A.L., Lisboa-Filho, P.N., Ortiz, W.A.: Temperature-dependent vortex motion in a square mesoscopic superconducting cylinder: Ginzburg-Landau calculations. Phys. Rev. B 74, 014512 (2006)

19. McLaughlin, D.W., Scott, A.C.: Perturbation analysis of fluxon dynamics. Phys. Rev. A 18(4), 16521680 (1978)

20. Tinkham, M.: Introduction to Superconductivity. McGraw-Hill, New York (1996)

21. Du, Q.: Global existence and uniqueness of solutions of the time-dependent Ginzburg-Landau model for superconductivity. Appl. Anal. 53(1), 2716-2723 (1994)

22. Zimmerman, W.B.J.: Multiphysics Modelling with Finite Element Methods. World Scientific, Singapore (2006)

23. COMSOL, Comsol Multiphysics Modeling Guide, Version 3.5a. www.comsol.com (2009) 